\title{
Biopsychosocial Concept is the Characteristic that Distinguishes the Practice of General Practitioner/Family Doctor
}

\section{Jose Luis Turabian ${ }^{1,2^{*}}$}

${ }^{1}$ Health Center Santa Maria de Benquerencia Toledo, Spain

${ }^{2}$ Regional Health Service of Castilla la Mancha (SESCAM), Toledo, Spain

*Corresponding author: Jose Luis Turabian, Health Center Santa Maria de Benquerencia Toledo, Spain, Tel: 34925154508; E-mail: jturabianf@hotmail.com

Received date: March 29, 2018; Accepted date: March 31, 2018; Published date: April 02, 2018

Copyright: @ 2018 Turabian JL, This is an open-access article distributed under the terms of the Creative Commons Attribution License, which permits unrestricted use, distribution, and reproduction in any medium, provided the original author and source are credited.

\section{Editorial}

In 1977 Engel, from the University of Rochester, proposed a paradigm capable of scientifically including the set of biological, psychological and social factors that determine health and disease [1]. From this biopsychosocial model (BPS), the disease is the result of a complex intertwining of symbolic networks and biological processes that unite the body, the self, the social and the environmental.

The BPS model derives from the general theory of systems. The essential points of this new vision are [2]: 1) There is no objective observer, independent and not involved, and so, the presence of the observer and his instruments disturb the phenomenon in a nondeterminable way; 2) There is no phenomenon in itself, the conjunction between the operator and the phenomenon produces a new condition in which phenomena appear that are products of both; 3) The causality of all phenomena is multiple, and so, causal relationships are complex and non-linear; 4) The concept of uncertainty is introduced; 5) It is not possible to fragment a phenomenon for its study, and thus the phenomena must always be taken as totalities; 6) Context must always be taken into account; and 7) The subjectivity of the observer is part of the process.

The BPS approach is to determine in each patient the specific proportion of the importance of each one of the biological, psychological and social factors, in each period of time. The BPS approach skills are crucial, and family doctor should be able at the same time to have a scientific, interactive and simultaneous approach to each problem from the biomedical, psychological, and social perspectives, and should be able to combine these perspectives in a panoramic vision to achieve a complete understanding. Family doctors know that the elements of the patient's personality and their social relationships have a great importance in the disease, and these elements are as evident and necessary as the biomedical aspects. The characteristic of family medicine is the understanding of the patient and his illness based not only on symptoms and signs, but on the psychological and social factors that relate the patient to its context.

The need to consider the psychological and social components in health care has been articulated for years. The dominant concept of "disease" assumes that it only reflects the biological (physiopathological) alterations, and the psychological and social factors are considered irrelevant: this is how stereotypes are adopted based on the pathology presented by the patient ("the osteoarthritic patient, the cardiac patient, the psychiatric patient, the bronchitic patient", etc.), and the person with the disease is not completely recognized, and therefore is not evaluated or treated in a comprehensive manner.
The BPS model identifies the biological, psychological and social factors as elements that are always present and with interrelated influences on health and illness. If the application of the scientific methodology discovers and allows to conclude that the so-called psycho-social factors participate in some grade in the process of determination of disease -in the origin, in the course or outcome- there is no reason to consider minor its importance in relation to that of other participating factors and award them secondary or distant positions. The basic difference of the biopsychosocial model is that it admits that psychological and social factors can influence and be influenced by the physiopathological process [3-6].

The disease in all cases is an alteration or dysfunction of communication relationships between actors and contexts (human beings, perceptions, environments, etc.). The intervention unit is the individual plus its context: relationships / connections / links between actors. Healing -the treatment, the intervention of the professionalbecomes possible through participation in the matrix of relationships with other people. The expression of individual symptoms, diagnosis, prognosis, course of evolution and treatment strategy are more related to certain contextual psychosocial factors than to the apparently specific pathological factors. It can be said that the disease arises from situations or contexts that do not allow the containment of symptoms. In addition, the disease does not refer only to organic symptoms, but to the set of experiences or sensations of the person in a series of contexts or situations. The common denominator of the disease is that it occurs or is born in situations and is a relational concept [7-8].

Somatic complaints without organic cause, what are called functional somatic syndromes, are frequent in family medicine [9]. Organic diseases frequently present psycho-social symptoms [10]. Thus, it is rare for the doctor to deny the impact of stress, the emotional and social factors in the existence and course of physical and mental illnesses, but this knowledge does not apply in the reality of the consultation. The same happens about the influence of the doctorpatient relationship. While physicians insist on the prevalence of somatizations in their practices, they generally continue to prescribe drugs for physical complaints as if they had a pathophysiological basis.

Medical training based on the biomedical model reinforces that professionals prefer physical explanations and are not receptive to the psychosocial factors of the disease. This biomedical approach tends to teach physicians to think of patients as carriers - reservoirs - of specific diseases rather than people with a high degree of BPS complexity. Another barrier to BPS training is the "technology imperative"; the technology seduces, the BPS model uses a "soft", "female" and "diffuse" technology, and its research areas cannot with the show of an NMR, or laser surgery, etc. In addition, conventional training means that after the faculty and years of residency, the doctor is harder, cold and 
Citation: Turabian JL (2018) Biopsychosocial Concept is the Characteristic that Distinguishes the Practice of General Practitioner/Family Doctor. J Gen Pract (Los Angel) 6: e122. doi:10.4172/2329-9126.1000e123

Page 2 of 2

cynical. Another barrier is the lack of models for the idealization of BPS work. Since most physicians are trained in the traditional method of the medical model, it tends to perpetuate itself.

Some practical tools of the biopsychosocial model to be applied in the tasks of consulting each day in family medicine are: the Interview (from the context to the symptom, decentralized and contextualized interview); the Contextualized Clinical History (list of problems, resources, and beliefs, list of "problems-force", grids of evaluation BPS of the patient, data collection of several actors, use of qualitative instruments in the taking of the clinical history); contextualized decisions (the value of continuity of care, experience, emotions, patient participation, strategy, etc.); other classical instruments of family medicine (family life cycle, genogram, stressful events / family crises; turning points and transitions), other classic instruments of community medicine (scales of social support, risk / morbidity and mortality groups), inventories of community resources (mutual help groups, etc.), relational capital, training protocols, inventory of patients-resources, and group consultations [8-12].

\section{References}

1. Engel GL (1977) The need for a new medical model: A challenge for biomedicine. Science 196: 129-36.

2. Balner H, DeVries MJ, Engel GL, Goodkin IK, LeShan L, et al. (1990) A new medical model: A challenge for biomedicine? Helen Dowling Institute for Biopsychosocial Medicine

3. Turabian JL, Franco BP (2006) The doctor with three heads. Aten Primaria 38: 570-3.
4. Turabian JL, Perez Franco B (2007) The biopsychosocial model and the "market of lemons". Aten Primaria 39: 329-30.

5. Turabian JL, Perez Franco B (2008) Do family doctors seem like turtles? Semergen 34: 373-4.

6. Turabián JL, Pérez Franco B (2007) Big mysteries. can you see the loch ness monster? The Biopsychosocial Model and Community Activities. Aten Primaria 39: 261-4.

7. Gannik DE (1995) Situational disease. Fam Pract 12: 202-6.

8. Turabián JL, Pérez Franco B (2008) Individual health care with community orientation - contextualized care: The figure is the background. Revista Clínica Electrónica en Atención Primaria.

9. Nakao M (2017) Somatic manifestation of distress: Clinical medicine, psychological, and public health perspectives. Biopsychosoc Med 11: 33.

10. Turabian JL (2017) Psychosocial factors and organic disease: Two sides of the same coin. Chronicle of Medicine and Surgery 1.1: 44-6.

11. Engel G (1980) The clinical appplication of the biopsychosocial model. Am J Psychiatry 137: 535-44.

12. Turabián JL, Pérez Franco B (2005) A way to make clinical pragmatism operative: Sistematization of the actuation of competent physicians. Med Clin (Bar) 124: 476 .

13. Kerns RD, Curley AL (1985) A biopsychosocial approach to illness and family: Neurological diseases across the life span. New York: John Wiley and Sons.

14. Turabián JL, Franco BP (2016) Turning Points and Transitions in the Health of the Patients: A Perspective from Family Medicine. J Family Med Community Health 3(4): 1087. 